\title{
Measurements of Immune Responses for Establishing Correlates of Vaccine Protection Against HIV
}

\author{
Wendy A. Burgers, Amapola Manrique, ${ }^{2}$ David Masopust, ${ }^{3}$ Lyle R. McKinnon, ${ }^{4}$ Matthew R. Reynolds, ${ }^{5}$ \\ Morgane Rolland, ${ }^{6}$ Catherine Blish, ${ }^{7}$ Gerald K. Chege, ${ }^{8}$ Rhonda Curran, ${ }^{9}$ William Fischer, ${ }^{10}$ \\ Carolina Herrera, ${ }^{11}$ and D. Noah Sather ${ }^{12}$
}

\begin{abstract}
Well-defined correlates of protective immunity are an essential component of rational vaccine development. Despite years of basic science and three HIV vaccine efficacy trials, correlates of immunological protection from HIV infection remain undefined. In December 2010, a meeting of scientists engaged in basic and translational work toward developing HIV-1 vaccines was convened. The goal of this meeting was to discuss current opportunities and optimal approaches for defining correlates of protection, both for ongoing and future HIV-1 vaccine candidates; specific efforts were made to engage young scientists. We discuss here the highlights from the meeting regarding the progress made and the way forward for a protective HIV-1 vaccine.
\end{abstract}

\section{Introduction}

E FFicAcious vaccines HAVE BEen developed against a range of human pathogens, often without an in depth understanding of correlates or immunological mechanisms of conferred protection. ${ }^{1-6}$ In contrast to past empirical approaches to vaccine design, there is hope that deciphering the underlying mechanisms of protection from HIV-1 infection or disease will facilitate HIV-1 vaccine design. Therefore, the Bill and Melinda Gates Foundation and the National Institutes of Health Office of AIDS Research convened a meeting of leading HIV-1 scientists to discuss current opportunities and strategies for identifying the correlates of protection associated with prevention of HIV-1 infection and/or disease. The ideas and data presented at this meeting summarized recent progress toward the measurement and identification of the correlates of protection against HIV-1, provided a forum for discussing the main challenges for the HIV vaccine field, and highlighted the renewed sense of optimism for developing a protective HIV-1 vaccine. Detailed in this report is a synopsis of the thoughts and recommendations put forth by the participants of the meeting.

Retrospective analysis of immune responses to efficacious vaccines that were developed empirically have yielded welldefined correlates of protection; for example, a certain titer and/or avidity of neutralizing antibody responses are necessary and sufficient for vaccine-induced protection from both smallpox and yellow fever. ${ }^{6,7}$ It is not clear whether similar types of immune responses would protect against HIV-1, and the rarity of populations demonstrating immunemediated protection from HIV-1 leaves us with no identifiable correlates to aim for in vaccine development. This workshop was entitled "Correlates of Vaccine Protection"; strictly speaking, however, correlates of vaccine protection can be

\footnotetext{
${ }^{1}$ Institute of Infectious Diseases and Molecular Medicine and Division of Medical Virology, University of Cape Town, Cape Town, South Africa.

${ }^{2}$ Global HIV Vaccine Enterprise, New York, New York

${ }^{3}$ Department of Microbiology, Center for Immunology, University of Minnesota Medical School, Minneapolis, Minnesota.

${ }^{4}$ Department of Medicine, University of Toronto, Toronto, Canada and Department of Medical Microbiology, University of Nairobi, Nairobi, Kenya.

${ }^{5}$ AIDS Vaccine Research Laboratory, University of Wisconsin-Madison, Madison, Wisconsin.

${ }^{6}$ U.S. Military HIV Research Program, Rockville, Maryland.

${ }^{7}$ Division of Infectious Diseases and Geographic Medicine, Department of Medicine, Stanford University, Stanford, California.

${ }^{8}$ Institute of Infectious Diseases and Molecular Medicine and Division of Medical Virology, University of Cape Town, Cape Town, South Africa.

${ }^{9}$ Institute of Nursing Research/School of Nursing, University of Ulster, Ulster, United Kingdom.

${ }^{10}$ Group T-6, Los Alamos National Laboratory, Los Alamos, New Mexico.

${ }^{11}$ Section of Infectious Diseases, Faculty of Medicine, St Mary's Campus, Imperial College, London, United Kingdom.

${ }^{12}$ Seattle Biomedical Research Institute, Seattle, Washington.
} 
defined only in the context of vaccine trials that show some level of protection. For HIV, only the RV144 trial $^{8}$ has shown modest protection from HIV-1 infection. Therefore, we use herein a broad definition of correlates of protection against HIV-1 infection and HIV-1 disease progression, which corresponds to the protective effects instilled by immune responses; an accompanying article focuses on statistical considerations regarding the definition of correlates of vaccine protection in the context of the RV144 trial (see Rolland and Gilbert). The identification of correlates of protection for HIV-1 relies on several factors: (1) the development of experimental vaccines, tested in humans and/or stringent animal models, that confer at least some degree of protection, (2) the ability to monitor relevant immunological responses that distinguish protected from susceptible individuals, and (3) the optimization of vaccine modalities that establish such correlates of protection.

\section{Ongoing Analysis of Completed Vaccine Trials}

At the outset of the meeting important updates were provided for studies on the correlates of protection associated with two HIV-1 vaccine regimens that have been tested in large-scale efficacy trials. The Phase III RV144 trial that took place in Thailand ${ }^{2}$ received particular attention. The vaccine regimen, consisting of prime-boost immunizations with ALVAC-HIV (a recombinant canarypox virus expressing HIV-1 Env, Gag, and Pol from clade E) and gp120 from clades B and E, had an overall protective effect of $31 \%$ against HIV acquisition. Although this modest degree of efficacy is insufficient for licensure, these results are exciting nevertheless, not only because they indicate that a protective HIV-1 vaccine may be possible, but also because they offer the first opportunity to identify correlates of vaccine protection in humans. However, it was also noted by the participants of the meeting that RV144 provides an example of the difficulty of defining the correlates of protection within the context of a large clinical trial. The sobering reality is that large efficacy trials take many years to complete and will often necessitate being followed-up by additional studies in other populations or risk groups (Fig. 1). For these reasons the breadth and depth of analyses being undertaken on samples from RV144 (Fig. 2) illustrate the enormous effort being undertaken to discover the correlates of vaccine protection to hasten the design of improved vaccines. Based on pilot studies, assays showing vaccine-induced signals that were reproducible and nonoverlapping were advanced for case-control studies.

As discussed at the meeting, it is also evident that analysis of past trials, even when no protective efficacy was achieved, can provide clues for future vaccines. For example, the trivalent Merck Adenovirus 5 (Ad5) (Gag, Pol, and Nef HIV-1 subtype B) homologous boost vaccine (tested in the Step trial) failed to confer protection or reduce viral load in vaccinees. ${ }^{9}$ However, analysis of HIV-1 sequences from breakthrough infections showed evidence of a "sieve effect," which showed that based on analysis of potential $\mathrm{CD}^{+} \mathrm{T}$ cell epitopes, sequences from vaccinees had a greater genetic distance to the vaccine sequence than sequences from placebo recipients. ${ }^{10}$ This suggests that vaccine-generated immune responses placed selective pressure on infecting viral strains. In addition, among those who became HIV infected but had protective HLA types, vaccinated individuals had lower viral setpoints compared to those receiving placebo. Thus, despite the lack of statistical efficacy and several unanswered questions, many lessons can be learnt from the Step trial and its sister trial in South Africa, Phambili. These experiences can provide information on the challenges of HIV genetic diversity and provide insights into the kind of immune responses required for protection. ${ }^{11,12}$

Following recent HIV-1 vaccine trial findings, the field has shifted focus to gain a more complete understanding of the complexities of immunological memory. Many components of the immune system may contribute to a successful anti-HIV response, and a multitude of assays with fine specificities are available, but technical and sampling limitations prevent testing of all immunological functions. Therefore, sample collection and assay selection must be prioritized toward the potentially most informative immune responses. How best to narrow down which immunological facets to focus on (and developing the assays to measure them) is an important priority for HIV-1 vaccine evaluation.

\section{Assay Considerations}

Given our current understanding of the heterogeneity of host immunity, much time was devoted at the meeting to current and potential measurement strategies that may provide insight into the correlates of protection. As such, screening for immunogenicity alone or standard flow cytometry methods that are often Th1 biased are no longer sufficient. This raises the issue of what to measure and how to link highly specialized assays typically applicable only in large academic/reference centers to more standard discovery research assays. Additional consideration must also be given to effectively measuring specific immune attributes using optimized, and ultimately validated, assays in multiple laboratories and settings.

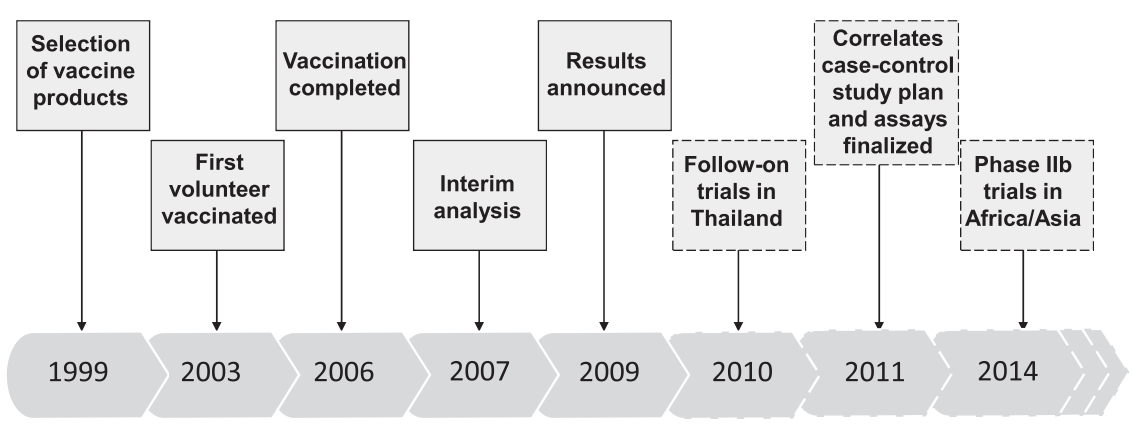

FIG. 1. Timeline of the RV144 trial. Dashed boxes indicate ongoing and planned studies and trials. 


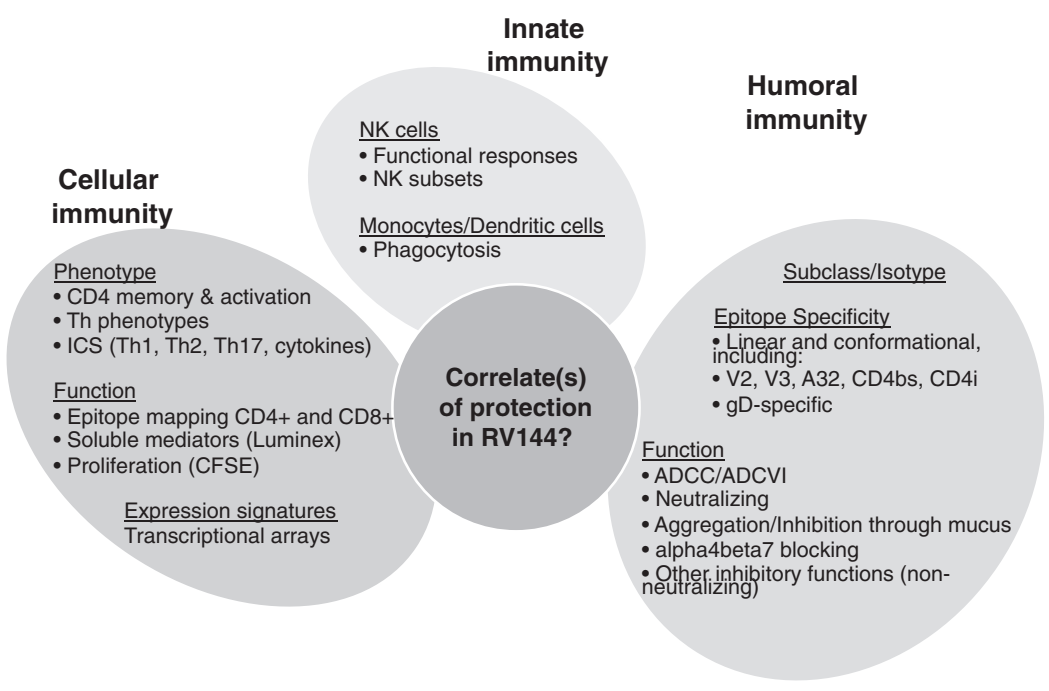

A point of emphasis at the meeting was that blood sampling may not provide a reliable surrogate for measuring responses in peripheral tissues. For instance, immune signatures identified in the peripheral blood may not accurately reflect events at mucosal surfaces and their potential associations with protection. At present, sampling of mucosal and lymphoid tissues is very limited in humans, and the availability of material greatly constrains the number of immunological assays that are feasible. To complement human vaccine studies, it is essential to use nonhuman primate (NHP) models, where timing of infection (challenge) is known and tissue sampling can be performed more frequently.

Another consideration presented at the meeting is that sampling strategies must yield sufficient material for planned assays with a margin for unanticipated needs. However, storage requirements (e.g., long-term freezer space) must be considered and sample collection must be acceptable to trial participants (i.e., not too unpleasant). The collected samples must retain high viability after processing and freezing (i.e., $>80 \%$ are viable after thaw). Sample collection is nontrivial, particularly for mucosal responses. Hence, there is a need for standardized procedures and routine QC of sample processing and storage, a factor that is as important as standardization of assays. Emphasis was placed on collecting samples as frequently as possible, to increase the probability of capturing the early events of infection and the corresponding anamnestic immune responses. This is particularly critical for understanding how the innate response may influence specific adaptive responses.

\section{$T$ cell immunity}

A topic of conversation at the meeting was the potential roles that different branches of the immune system may play in the development of a protective HIV-1 vaccine. Considerable evidence suggests that $\mathrm{T}$ cells will be a critical component of effective HIV-1 protective immunity. CD8 ${ }^{+} \mathrm{T}$ cells can contribute to the control of primary HIV-1 infection ${ }^{13,14}$ and vaccines inducing these cells in NHP models have resulted in impressive reductions in viral setpoint ${ }^{15}$ and vaccine-induced responses may even be able to control the infection. ${ }^{16,17}$ There has been considerable debate about the risks associated with vaccine-induced $\mathrm{CD}^{+}{ }^{+} \mathrm{T}$ cell responses: given that $\mathrm{CD} 4^{+} \mathrm{T}$ cells represent the major target of HIV-1, they could be implicated in enhanced susceptibility to infection. However, $\mathrm{CD}^{+}{ }^{+} \mathrm{T}$ cells are critical for inducing functional $\mathrm{CD}^{+} \mathrm{T}$ cell memory and long-lived high-affinity antibody responses, and may thus be a prerequisite for any vaccine aimed to establish long-lived immunological memory. $\mathrm{CD}^{+} \mathrm{T}$ cells that produce $\beta$-chemokines such as $\mathrm{MIP} 1 \alpha$ and $\beta$ may provide immunity while being less susceptible to HIV infection. ${ }^{18}$

How to induce the "right" kind of $\mathrm{CD}^{+}{ }^{+} \mathrm{T}$ cell responses, and how these relate to other immune functions, remains unknown and was a topic of discussion at the meeting. Two parameters that may influence $\mathrm{CD}^{+} \mathrm{T}$ cell quality are the length of antigen exposure and the cytokine milieu during priming. A subset of $\mathrm{CD}^{+}{ }^{+} \mathrm{T}$ cells called follicular helper $\mathrm{T}$ cells (TFH) has been the focus of recent attention. TFH provide essential help for long-lived humoral immunity and are predominantly located within lymph nodes, in close association with B cells. Given the short duration and limited functional capacity of specific antibodies induced by HIV vaccines to date, a better understanding of potential TFH induction by HIV vaccines may be an important advance toward eliciting protective responses.

It is reasonable to conclude that both $\mathrm{CD}^{+}$and $\mathrm{CD}^{+} \mathrm{T}$ cell responses will have important roles in vaccine-induced protection from HIV. However, although measuring different functional and phenotypic T cell subsets is technically possible with advanced flow cytometry, performing these assays in vaccine trials and interpreting the meaning of the data can be challenging. Memory $\mathrm{T}$ cells exhibit tremendous functional heterogeneity; the specific functions that correlate with protective immunity remain unclear. Moreover, important responses might be epitope specific and HLA restricted. Time, money, and sampling limitations make it impossible to directly assess all potentially relevant functions in clinical trials. One way around this may be to identify reliable surrogates of important immune qualities that are cumbersome to measure. For example, other aspects of memory $\mathrm{CD} 8^{+} \mathrm{T}$ cell quality might be represented by a simpler assay, such as functional avidity, cytokine expression, or expression of certain markers (e.g., expression of CD27 often correlates with proliferative potential). 
The participants at the meeting believed that memory $\mathrm{CD}^{+} \mathrm{T}$ cells will likely need to be elicited at the point of viral entry or initial viral replication, which most often includes the mucosal gastrointestinal and genital tracts. ${ }^{19,20} \mathrm{~T}_{\mathrm{FH}} \mathrm{CD} 4^{+} \mathrm{T}$ cells, which provide essential help for long-lived humoral immunity, are predominantly located within lymphoid tissue. Developing improved assays for accurately measuring mucosal T cell responses, or discovering surrogates in blood for mucosal responses, is a critical goal.

\section{Antibody immunity}

HIV-1-specific antibodies have a number of potential roles in the search for the correlates of HIV-1 protection and, therefore, were a major topic of discussion at the meeting. Neutralizing antibodies are able to confer sterilizing immunity if present prior to simian-human immunodeficiency virus (SHIV) challenge in NHP models. ${ }^{21-23}$ However, how antibodies capable of neutralizing diverse strains of HIV can be induced by vaccination remains unknown. Furthermore, there is a growing body of evidence suggesting that nonneutralizing activities may play a role in protective immunity, such as antibodydependent cellular cytotoxicity (ADCC) ${ }^{24}$ and antibodydependent cellular viral inhibition (ADCVI). ${ }^{25,26}$ In addition to peripheral blood, it may also be necessary to survey antibody responses at mucosal sites, and in the saliva, urine, and stool. Continual refinement of current assays to improve biological relevance, innovation of new assays, as well as expanded and improved collection techniques will accelerate this effort.

Anti-Env antibody responses induced by HIV vaccine candidates are predominantly short-lived, however, there are cases in which durable vaccine-elicited antibodies were detected. ${ }^{27-29}$ Long-lasting B cell memory has been described during natural HIV infection, although functional defects have been observed. ${ }^{30}$ This is in contrast with many live replicating vaccines and natural infections that are cleared, where B cell immunity is life-long. ${ }^{5,7}$ To achieve long-lasting antibody responses to HIV-1, a key priority is to develop improved Env immunogens, adjuvants, and immunization protocols that will promote B cell maturation, somatic hypermutation, and rapid B cell memory recall. The possibility that this will require live replicating vaccine vectors should be explored. In addition to the induction of long-lived responses, a vaccine strategy would probably need to address the extensive affinity maturation that broadly neutralizing antibody responses to HIV-1 demonstrate. If inducing broadly neutralizing antibodies with rare specificities is a prerequisite that cannot be overcome by a single vaccine, it is possible that repeated administration, long-term exposure of the antigen, or novel immunogen strategies that target the germlines of broadly neutralizing antibodies may be needed. There is a pressing need for vectors and/or antigens capable of improving upon and inducing stronger antibody immunity and accompanying $\mathrm{CD}^{+}{ }^{+} \mathrm{T}$ cell help.

As with T cells, more aggressive tissue sampling may prove important. In addition to peripheral blood mononuclear cells (PBMC), gut-associated lymphoid tissue and bone marrow aspirates permit the analysis of antibody production through the generation of monoclonal antibodies. The "antibodyome" enables fine mapping of humoral responses, analysis of memory/long-lived plasma cells, and analysis of antibody production at mucosal sites.

\section{What Information Should NHP Studies and Vaccine Clinical Research Provide?}

Compared to human clinical trials, NHP studies allow for sampling relevant tissue compartments, particularly early after challenge and / or infection, for in-depth investigations of early virus-host interactions. Such studies in the mucosal compartment are particularly important, as they could lead to a better understanding of viral acquisition, transmission across the different mucosal tissues, and vaccine-specific mechanisms associated with protection. Comprehensive NHP studies can compare and contrast immune responses located in peripheral blood and divergent mucosal tissues in ways that are impractical or impossible in humans. To accelerate this effort, NHP vaccine studies should incorporate indepth analyses of tissue-resident immune responses as well as exploit new technologies (e.g., microarrays, single cell technologies). A recurring topic during the meeting was the need to pair vaccines progressing to phase IIb or phase III trials in humans with NHP studies of a directly comparable vaccine regimen. The goal of such parallel studies would be, in part, to determine the effectiveness of the vaccine regimens in preclinical settings and ascertain the anatomical location and longevity of vaccine-induced immune responses that correlate with protection. If protection is demonstrated in humans, studies that correlate the human and NHP responses with protection would dramatically improve the utility of NHP as models for human HIV-1 infection. Such studies in NHP should be an integral complementary approach to the identification of the immune correlates of protection in humans.

A common misperception brought forward at the meeting is that we can rely only on a single NHP model of HIV-1 infection. The field has now developed multiple models based on different macaque species, challenge viruses, and challenge routes. Any combination of these factors can constitute a distinct model. The key is to choose the appropriate NHP model that closely recapitulates the relevant features of HIV biology, transmission, and pathogenesis. For example, recent refinements of SIV challenge models with repeated limitingdose virus challenges effectively replicate the transmission of a single virus across mucosal surfaces, as typically seen in sexual HIV-1 transmission in humans. ${ }^{17}$

Studies in NHP also present difficulties and limitations. Various factors can influence the outcome of vaccine and correlates studies in NHP, including species, age, MHC composition, and innate restriction factor (e.g., TRIM $5 \alpha$ ) genotype. A number of SIV and SHIV challenge stocks exist, and the virus and challenge model should be carefully selected to yield the most significant information. As discussed at the meeting there are additional country-specific challenges that hinder researchers' ability to perform NHP studies, such as differences in ethical guidelines and cost limitations. Collectively, NHP models provide unique advantages for the study of correlates of immune protection, even if scientific and structural hurdles must still be overcome. Moving forward will require more focused communication between NHP and human clinical researchers to narrow down priorities for future research.

\section{The Search for Correlates: Where to Go from Here?}

Successful vaccines such as smallpox, yellow fever, and measles are all known to confer long-lived protection even if 
the exact duration of the protection has not been clearly established. For example, boosting is still recommended for the yellow fever vaccine, even though protection appears to last longer than the 10 year cycle of yellow fever vaccinations, with studies suggesting up to $30-35$ years of protection. ${ }^{31}$ In these examples, it is reasonable to believe this immunity is mediated by neutralizing antibodies. In contrast, toxoid subunit vaccines produce antibody titers with a much shorter half-life; for example, less than half of hepatitis B vaccinees retain antibody responses after 23 years. ${ }^{5,32}$ Data presented at the meeting suggest that HIV vaccine responses so far appear to be even shorter-lived ( $<1$ year). These results illustrate that not all vaccine modalities lead to long-lived immunity, and that the "quantity" of immunological memory, in addition to quality, may be a critical determinant of protection. While correlates of protective immunity may differ between HIV and other pathogens, successful vaccines provide a benchmark for what is possible, and may be necessary, for protection. Moving forward, it will be critical to assess the dynamic heterogeneity of HIV-specific responses induced by vaccination. Because immune responses are extremely complex, and the potential correlates of protective immunity to HIV are unknown, this poses a formidable challenge given that it is not feasible to measure all of the possible responses that may be induced by a vaccine.

\section{New trial designs to streamline vaccine development}

One major theme from completed efficacy trials discussed at the meeting is the unexpected nature of the results obtained. One conclusion from this observation is that we need to test more concepts, ideally incorporating mechanisms to identify correlates as these trials progress. To date, although many phase I/II HIV-1 vaccine clinical trials have been conducted, only three vaccine candidates have been tested in large-scale efficacy trials. After 25 years of HIV vaccine research, it seems that vaccine trials could be restructured less conservatively, such is the new concept that is now being proposed for randomized, blinded, phase IIb trials: "adaptive clinical trial designs." ${ }^{\prime 33,34}$ Adaptive clinical trials are able to test multiple candidates simultaneously and to compare them to a single placebo group. This type of trial may make it possible to prioritize the most promising new candidate HIV vaccines for further evaluation, and to eliminate rapidly those that show negligible or negative effects. The drawback of adaptive clinical trial designs is reduced statistical power. However, the parallel structure of adaptive clinical trial designs could provide more rapid evaluation, and iterative improvement, of new vaccine concepts and methodologies than is possible with traditional trial designs.

\section{New antigens and vectors}

In past clinical trials, laboratory strains or sequences corresponding to circulating viruses have been used to generate vaccine immunogens (as proteins or as inserts expressed in suitable vectors). These products represent suboptimal choices given the high genetic diversity of HIV-1 and its propensity to mutate, as any infecting virus will be as different from such antigens as from any other circulating strain. Recent designs have been engineered to take into account the considerable variability of HIV-1. One approach considered at the meeting, mosaic antigens, uses computer algorithms to design antigens maximizing the coverage of potential $\mathrm{T}$ cell epitopes commonly occurring in natural HIV strains worldwide. ${ }^{35-37}$ Variability-inclusive antigens seek to cover the most common variant peptides found in HIV-1 viruses and thereby to possibly block common escape pathways.

Designing improved HIV antigens is only part of the effort needed to produce more effective HIV vaccines. Further research needs to be performed into how to optimally present these antigens to the immune system to induce effective, longlasting responses. An aspect of the RV144 trial is the apparent brief durability of the already modest protection. Env antigens delivered with improved adjuvants and vectors could induce more effective and longer-lived antibody responses. Similarly, knowledge concerning what constitutes an effective $\mathrm{T}$ cell response in terms of breadth, quantity, quality, and anatomical location of $\mathrm{T}$ cells should dictate what immunization strategies and vectors need to be incorporated into HIV vaccines. For longevity of both antibody and T cell responses, live-replicating vectors may be required; the safety of these vectors, however, is a concern in humans. Nevertheless, the effectiveness of rhesus cytomegalovirus (rhCMV) vectors, which establish persistent infection in macaques, in effectively controlling infection revealed the tantalizing potential of this approach. Therefore, there was support at the meeting for additional research into improved adjuvants and vectors to more effectively induce long-lived immune responses at relevant anatomical locations.

\section{Other considerations for future HIV vaccine trials}

An important point presented at the meeting is the need for including behavioral and social scientists when designing protocols for clinical trials. In recent microbicide and PrEP trials, combinations of self-reported adherence and biological measures corresponded to higher rates of protection. ${ }^{38,39}$ The increasing availability of mobile phone devices in resourcelimited settings provides new ways to obtain sensitive behavioral data on sexual activity and drug adherence.

Vaccine modalities may not be universally effective against all routes of transmission; immune correlates may differ between routes of transmission, hence between populations. Thus, the risk group in which a trial is conducted may affect the outcome of an efficacy trial. It is possible that trials conducted in low-risk populations might afford different results if conducted in higher-risk groups, and data are currently insufficient for modeling how results might differ between risk groups. It may be critical to focus on concentrated epidemics, and target particular high-risk groups for which the cost effectiveness of, and need for, a vaccine is highest.

Recent advances in HIV prevention research are considered for future HIV vaccine trial designs. The results from trials of male circumcision, ${ }^{40-42}$ a vaginal microbicide, ${ }^{43}$ and oral preexposure prophylaxis in men who have sex with men ${ }^{44}$ showed that these interventions can effectively reduce HIV-1 transmission. Some of these interventions are currently being implemented, or are imminent; others need confirmatory trials, and whether vaccines and PrEP can act synergistically is an open question. ${ }^{45}$ In addition, vaccine clinical trial designs will soon need to take into account the ethical imperative to provide access to effective prevention modalities for all vaccine trial participants. This will undoubtedly limit the overall power of vaccine trials, and community engagement will be required in order to recruit enough trial participants. 


\section{Sharing data}

A point of emphasis at the meeting was that the search for correlates requires a strengthened commitment to make data from vaccine trials available to the global scientific community. This could include taking advantage of advances in technology to develop central repositories, user-friendly web portals, and tools to facilitate annotation and management of data. Efforts should also be directed toward creating regulatory agreements that would permit timely sharing of data among research groups. The value of having the data from previous studies available to the scientific community was exemplified by reanalysis of the AIDSVAX trial (VAX004), which found good antibody responses in a portion of protected vaccinees ${ }^{46}$ (B. Korber, unpublished observation).

\section{Conclusions}

In conclusion, generating a vaccine against HIV-1 has proven far more difficult than for many other pathogens, likely a result of unique features of HIV-1 immunity and pathogenesis. The motivation for defining correlates of immune protection is clear-to speed the identification of promising candidates that can then be evaluated in large-scale efficacy trials. The search for correlates of protection requires a multifaceted approach that will take into account our understanding of immunological memory and of HIV-1 disease containment in elite controllers and other groups, how we measure immune responses, the usefulness of NHP models, and greater issues facing the objective of achieving vaccinemediated protection against HIV-1 in humans.

This meeting addressed how ongoing analyses of previous vaccine clinical trials can help the search for correlates. These trial results have highlighted the need for more durable immune responses, and vaccine improvement will definitely require a better understanding of the mechanisms behind the longevity of immune responses. Recent studies have also begun to reveal the complexity and heterogeneity of effector and memory responses, pointing to the need for new assays that reflect current knowledge of the critical parameters of po-

Table 1. Key Recommendations to Advance the SEARCh for Correlates of Protection

Fully exploiting NHP models, e.g., intensive mucosal and lymphoid tissue sampling and analyses

Parallel preclinical NHP and clinical studies with advanced vaccine candidates, and greater interaction and communication between NHP and clinical researchers

Application of new and technologically advanced methods in vaccine studies (microarrays, single cell assays, etc.) to detect vaccine-induced responses

Substantial effort to develop surrogate assays for mucosal responses from blood

Advancing basic research into understanding the nature and timing of $\mathrm{CD}^{+}$help for long-lived $\mathrm{T}$ cell memory and antibody affinity maturation for vaccine-induced responses

Developing the infrastructure for storing and sharing samples and data from vaccine trials

More tests of concept clinical trials with flexible design to facilitate the evaluation of new products

NHP, nonhuman primate. tentially protective immune responses. The approach taken to evaluate past trials has fostered collaborations between many laboratories and will certainly result in increased comparability of results and improved standardization of assays across laboratories. New insights offered by reanalysis of past trials illustrate that a commitment should be made to create an infrastructure that would allow the rapid sharing of data with the global scientific community, both in terms of portals or repositories and regulatory agreements.

Adaptive clinical trial designs appear to be an important strategy to accelerate the pace of vaccine trials and to permit the evaluation of multiple vaccine candidates. There is a clear need to construct an agenda that integrates basic, preclinical, and clinical research and takes advantage of optimized NHP models. For future vaccine clinical trials, it is imperative that efforts are taken to obtain as many samples as possible for downstream analyses. Recent major progress with other prevention methodologies highlights the urgent need for concerted efforts by HIV vaccine researchers to develop an effective vaccine. We summarize key recommendations to advance the search for correlates of protection in Table 1.

\section{Acknowledgments}

We would like to acknowledge the assistance of Galit Alter, Guido Ferrari, Jonathan Fuchs, Clive Gray, Rick Koup, Penny Moore, and Mark Slifka for running the workshop on Correlates of Vaccine Protection, and guidance in preparing the manuscript. Many thanks to the presenters at this workshop, Galit Alter, Victor Appay, Linda-Gail Bekker, Jake Estes, Nicole Frahm, Genoveffa Franchini, Paul Goepfert, Nilu Goonetilleke, Barton Haynes, R. Paul Johnson, Chitraporn Karnasuta, Brandon Keele, Anthony Kelleher, Jerome Kim, Bette Korber, Jeff Lifson, Julie McElrath, Jim Mullins, Mario Roederer, Quentin Sattentau, Robin Shattock, and Georgia Tomaras. Finally, we thank the Global HIV Vaccine Enterprise and the OCTAVE Project for funding and organizing the Correlates of Vaccine Protection workshop for Young and Early Career Investigators; the Bill and Melinda Gates Foundation and the NIH Office for AIDS Research for funding the succeeding Correlates of Vaccine Protection meeting and Bonnie Mathieson, José Esparza, and Alan Bernstein for their contributions to the Scientific Committee of the meeting. W.A.B., A.M., D.M., L.R.M., M.R.R., and M.R. are the primary contributors to the article.

\section{Author Disclosure Statement}

No competing financial interests exist.

\section{References}

1. Lambert PH, Liu M, and Siegrist CA: Can successful vaccines teach us how to induce efficient protective immune responses? Nat Med 2005;11:S54-62.

2. Plotkin SA: Correlates of protection induced by vaccination. Clin Vaccine Immunol 2010;17:1055-1065.

3. Plotkin SA: Vaccines: Correlates of vaccine-induced immunity. Clin Infect Dis 2008;47:401-409.

4. Moxon ER, Das P, Greenwood B, et al.: A call to action for the new decade of vaccines. Lancet 2011;378:298-302.

5. Plotkin SA: Vaccines: Past, present and future. Nat Med 2005;11:S5-11. 
6. Amanna IJ, Messaoudi I, and Slifka MK: Protective immunity following vaccination: How is it defined? Hum Vaccine 2008;4:316-319.

7. Hammarlund E, Lewis MW, Hansen SG, et al.: Duration of antiviral immunity after smallpox vaccination. Nat Med 2003;9:1131-1137.

8. Rerks-Ngarm S, Pitisuttithum P, Nitayaphan S, et al.: Vaccination with ALVAC and AIDSVAX to prevent HIV-1 infection in Thailand. N Engl J Med 2009;361:2209-2220.

9. Buchbinder SP, Mehrotra DV, Duerr A, et al.: Efficacy assessment of a cell-mediated immunity HIV-1 vaccine (the Step Study): A double-blind, randomised, placebocontrolled, test-of-concept trial. Lancet 2008;372:1881-1893.

10. Rolland M, Tovanabutra S, deCamp AC, et al.: Genetic impact of vaccination on breakthrough HIV-1 sequences from the STEP trial. Nat Med 2011;17:366-371.

11. McElrath MJ, De Rosa SC, Moodie Z, et al.: HIV-1 vaccineinduced immunity in the test-of-concept Step Study: A casecohort analysis. Lancet 2008;372:1894-1905.

12. Gray GE, Allen M, Moodie Z, et al.: Safety and efficacy of the HVTN 503/Phambili study of a clade-B-based HIV-1 vaccine in South Africa: A double-blind, randomised, placebo-controlled test-of-concept phase 2b study. Lancet Infect Dis 2011;11:507-515.

13. Koup RA, Safrit JT, Cao Y, et al.: Temporal association of cellular immune responses with the initial control of viremia in primary human immunodeficiency virus type 1 syndrome. J Virol 1994;68:4650-4655.

14. Borrow P, Lewicki H, Hahn BH, Shaw GM, and Oldstone MB: Virus-specific CD8 + cytotoxic T-lymphocyte activity associated with control of viremia in primary human immunodeficiency virus type 1 infection. J Virol 1994;68:6103-6110.

15. Watkins DI, Burton DR, Kallas EG, Moore JP, and Koff WC: Nonhuman primate models and the failure of the Merck HIV-1 vaccine in humans. Nat Med 2008;14:617-621.

16. Hansen SG, Vieville C, Whizin N, et al.: Effector memory $\mathrm{T}$ cell responses are associated with protection of rhesus monkeys from mucosal simian immunodeficiency virus challenge. Nat Med 2009;15:293-299.

17. Keele BF, Li H, Learn GH, et al.: Low-dose rectal inoculation of rhesus macaques by SIVsmE660 or SIVmac251 racapitulates human mucosal infection by HIV-1. J Exp Med 2009; 206:1117-134.

18. Casazza JP, Brenchley JM, Hill BJ, et al.: Autocrine production of beta-chemokines protects CMV-specific CD4 T cells from HIV infection. PLoS Pathog 2009;5:e1000646.

19. Haynes BF and Shattock RJ: Critical issues in mucosal immunity for HIV-1 vaccine development. J Allergy Clin Immunol 2008;122:3-9; quiz 10-1.

20. Critchfield JW, Young DH, Hayes TL, et al.: Magnitude and complexity of rectal mucosa HIV-1-specific CD8 + T-cell responses during chronic infection reflect clinical status. PLoS One 2008;3:e3577.

21. Shibata R, Igarashi T, Haigwood N, et al.: Neutralizing antibody directed against the HIV-1 envelope glycoprotein can completely block HIV-1/SIV chimeric virus infections of macaque monkeys. Nat Med 1999;5:204-210.

22. Baba TW, Liska V, Hofmann-Lehmann $R$, et al.: Human neutralizing monoclonal antibodies of the IgG1 subtype protect against mucosal simian-human immunodeficiency virus infection. Nat Med 2000;6:200-206.

23. Mascola JR, Stiegler G, VanCott TC, et al.: Protection of macaques against vaginal transmission of a pathogenic HIV1 /SIV chimeric virus by passive infusion of neutralizing antibodies. Nat Med 2000;6:207-210.
24. Florese RH, Demberg $\mathrm{T}$, Xiao $\mathrm{P}$, et al.: Contribution of nonneutralizing vaccine-elicited antibody activities to improved protective efficacy in rhesus macaques immunized with Tat/Env compared with multigenic vaccines. J Immunol 2009;182:3718-3727.

25. Hessell AJ, Hangartner L, Hunter M, et al.: Fc receptor but not complement binding is important in antibody protection against HIV. Nature 2007;449:101-104.

26. Xiao P, Zhao J, Patterson LJ, et al.: Multiple vaccine-elicited nonneutralizing antienvelope antibody activities contribute to protective efficacy by reducing both acute and chronic viremia following simian/human immunodeficiency virus SHIV89.6P challenge in rhesus macaques. J Virol 2010;84: 7161-7173.

27. Goepfert PA, Tomaras GD, Horton H, et al.: Durable HIV-1 antibody and T-cell responses elicited by an adjuvanted multi-protein recombinant vaccine in uninfected human volunteers. Vaccine 2007;25:510-518.

28. Evans TG, Frey S, Israel H, et al.: Long-term memory B-cell responses in recipients of candidate human immunodeficiency virus type 1 vaccines. Vaccine 2004;22:2626-2630.

29. Vaine M, Wang S, Liu Q, et al.: Profiles of human serum antibody responses elicited by three leading HIV vaccines focusing on the induction of Env-specific antibodies. PLoS One 2010;5:e13916.

30. Moir S and Fauci AS: B cells in HIV infection and disease. Nat Rev Immunol 2009;9:235-245.

31. Poland JD, Calisher $\mathrm{CH}$, Monath TP, Downs WG, and Murphy K: Persistence of neutralizing antibody 30-35 years after immunization with 17D yellow fever vaccine. Bull World Health Organ 1981;59:895-900.

32. Wu Q, Zhuang GH, Wang XL, Wang LR, Li N, and Zhang M: Antibody levels and immune memory 23 years after primary plasma-derived hepatitis B vaccination: Results of a randomized placebo-controlled trial cohort from China where endemicity is high. Vaccine 2011;29:2302-2307.

33. Koup RA, Graham BS, and Douek DC: The quest for a T cellbased immune correlate of protection against HIV: A story of trials and errors. Nat Rev Immunol 2011;11:65-70.

34. Corey L, Nabel GJ, Dieffenbach C, et al.: HIV-1 vaccines and adaptive trial designs. Sci Transl Med 2011;3:79ps13.

35. Fischer W, Perkins S, Theiler J, et al.: Polyvalent vaccines for optimal coverage of potential T-cell epitopes in global HIV-1 variants. Nat Med 2007;13:100-106.

36. Barouch DH, O'Brien KL, Simmons NL, et al.: Mosaic HIV-1 vaccines expand the breadth and depth of cellular immune responses in rhesus monkeys. Nat Med 2010;16: 319-323.

37. Santra S, Liao HX, Zhang $\mathrm{R}$, et al.: Mosaic vaccines elicit $\mathrm{CD} 8+\mathrm{T}$ lymphocyte responses that confer enhanced immune coverage of diverse HIV strains in monkeys. Nat Med 2010;16:324-328.

38. Buchbinder SP and Liu A: Pre-exposure prophylaxis and the promise of combination prevention approaches. AIDS Behav 2011;15(Suppl 1):S72-9.

39. Veronese F, Anton P, Fletcher CV, et al.: Implications of HIV PrEP trials results. AIDS Res Hum Retroviruses 2011;27:8190.

40. Bailey RC, Moses S, Parker CB, et al.: Male circumcision for HIV prevention in young men in Kisumu, Kenya: A randomised controlled trial. Lancet 2007;369:643-656.

41. Gray RH, Kigozi G, Serwadda D, et al.: Male circumcision for HIV prevention in men in Rakai, Uganda: A randomised trial. Lancet 2007;369:657-666. 
42. Auvert B, Taljaard D, Lagarde E, Sobngwi-Tambekou J, Sitta $\mathrm{R}$, and Puren A: Randomized, controlled intervention trial of male circumcision for reduction of HIV infection risk: The ANRS 1265 Trial. PLoS Med 2005;2:e298.

43. Abdool Karim Q, Abdool Karim SS, Frohlich JA, et al.: Effectiveness and safety of tenofovir gel, an antiretroviral microbicide, for the prevention of HIV infection in women. Science 2010;329:1168-1174.

44. Grant RM, Lama JR, Anderson PL, et al.: Preexposure chemoprophylaxis for HIV prevention in men who have sex with men. N Engl J Med 2010;363:2587-2599.

45. Excler JL, Rida W, Priddy F, et al.: AIDS vaccines and preexposure prophylaxis: Is synergy possible? AIDS Res Hum Retroviruses 2011;27:669-680.
46. Gilbert $\mathrm{P}$, Wang $\mathrm{M}$, Wrin T, et al.: Magnitude and breadth of a nonprotective neutralizing antibody response in an efficacy trial of a candidate HIV-1 gp120 vaccine. J Infect Dis 2010;202:595-605.

Address correspondence to: David Masopust Department of Microbiology Center for Immunology University of Minnesota Medical School 2641 Campus Delivery, 2101 6th Street, South East Minneapolis, Minnesota 55455

E-mail: masopust@umn.edu 